\title{
Crossed four-bar mechanism for improved prosthetic grasp
}

\author{
Issa A. Ramirez, MS; ${ }^{1}$ Craig P. Lusk, PhD; ${ }^{1 *}$ Rajiv Dubey, PhD; ${ }^{1}$ M. Jason Highsmith, DPT, CP; ${ }^{2}$ Murray E. \\ Maitland, PhD, PT $^{3}$ \\ ${ }^{1}$ Department of Mechanical Engineering and ${ }^{2}$ School of Physical Therapy and Rehabilitation Sciences, University of \\ South Florida, Tampa, FL; ${ }^{3}$ Department of Rehabilitation Medicine, University of Washington, Seattle, WA
}

\begin{abstract}
Passive linkages were developed to improve grasp functionality and minimize a prosthetic terminal device's number of user-controlled inputs. The linkages act to stabilize grasped objects and substitute for the palp of normal anatomical fingers. The Southampton Hand Assessment Procedure was used to compare the normal anatomical hand, this prototype, and a commercially available (Hosmer) hook. In testing, prosthetic terminal devices took three times as long as the normal anatomical hand to perform tasks. Nevertheless, heavyweight power and spherical grasps were improved with the use of the new mechanism compared with the commercial hook. Conversely, precision grasps were worsened because of the lack of a high-friction surface on the distal end of the prototype.
\end{abstract}

Key words: amputation, four-bar mechanism, grasp, hook, polycentric, prehension, prosthetic hand, SHAP, terminal device, upper-limb prosthetics.

\section{INTRODUCTION}

Prosthetic hands (terminal devices) should be simple for persons with amputations to use and should contribute to their performance in grasping tasks. Different terminal devices are beneficial to activities of daily living (ADL) to varying degrees. Some terminal devices are specific to a narrow scope of applications, e.g., kayaking [1] or weight lifting [2], while others, like conventional hook terminal devices, can be used across a wide variety of activities. Like any tool, a terminal device might enhance performance in some situations but be neutral or detrimental in others.
The purpose of this study was to measure the enhancement or detriment caused by the introduction of passive four-bar mechanisms at the point of contact between a hook terminal device and a grasped object.

\section{DESCRIPTION OF FOUR-BAR MECHANISM}

Normal anatomical human hands have a compliant surface on the fingers: the finger pulp that is the fingerobject interface. The interface for typical hooks is a rigid, nonadaptive surface. In our prototype, we used a crossed four-bar linkage system as a rigid adaptive interface. The links are labeled $\mathrm{L}_{1}, \mathrm{~L}_{2}, \mathrm{~L}_{3}$, and $\mathrm{L}_{4}$ (Figure 1) and their respective lengths are given in Table 1 . $L_{3}$, the grasp surface, was coated with a high-friction material.

We analyzed the stability of the four-bar mechanism by using the principle of virtual work in Ramirez et al. [3]. In this analysis, we found that the fingertip mechanism adapts to the location and direction of the applied

Abbreviations: $\mathrm{ADL}=$ activities of daily living, $\mathrm{IOF}=$ Index of Functionality, SHAP = Southampton Hand Assessment Procedure.

*Address all correspondence to Craig P. Lusk, PhD; Department of Mechanical Engineering, University of South Florida, 4202 East Fowler Avenue, ENB 118, Tampa, FL 33620-5350; 813-974-1394; fax: 813-974-3539.

Email: clusk2@eng.usf.edu

DOI:10.1682/JRRD.2009.01.0004 


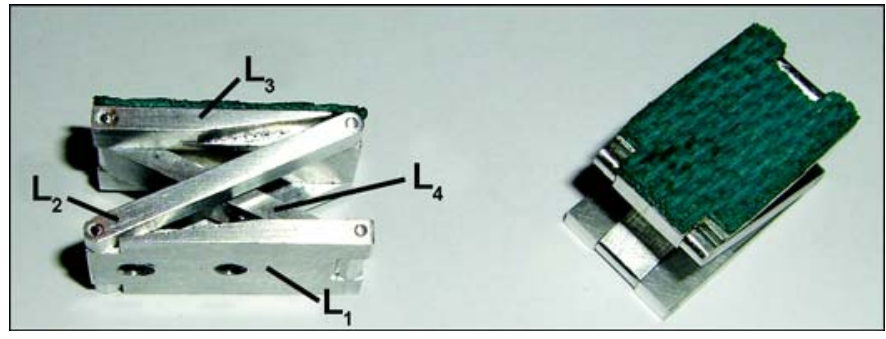

Figure 1.

Side and top views of crossed four-bar linkages $\left(\mathrm{L}_{1}-\mathrm{L}_{4}\right)$.

force, achieving a stable $\mathrm{L}_{3}$ orientation for a wide variety of contact points and loading directions through which the force might be applied, and that the stability of the link is indifferent to the magnitude of the force applied to it (assuming that the force does not damage/deform the mechanism).

\section{METHODS}

\section{Subjects}

The study was a within-subject repeated-measures design. The protocol was approved by the University of South Florida Institutional Review Board. As in Lake [4], the subjects in our study did not have amputations; thus, they could complete the protocol under three different conditions: normal anatomical hand, hook prosthesis, and four-bar linkage prosthesis. The subjects' use of prosthetic hooks was accomplished by means of a pseudoprosthesis that allows subjects without amputations to open and close a prosthetic hand located just past their closed fist while wearing the same body-power harness worn by persons with amputations. In using subjects without amputations, we could avoid significant withinsubject differences in upper-limb amputation level and function. The effect, studied by Lake, of a subject's previous experience with a specific terminal device could also be minimized [4].

For this research, 10 subjects (8 males and 2 females) between the ages of 18 and 25 were selected and proper informed consent was obtained. Personal history was collected from each person, including age at time of the assessment, race/ethnicity, and sex. The selected age interval was convenient in the university setting: a normative database has been established as the benchmark of normal hand function in that age range [5] and the age range reduces potentially significant variations within the
Table 1.

Geometric parameters (length in centimeters) of prototype crossed four-bar-linkage system. $\mathrm{L}=$ link.

\begin{tabular}{ccccc}
\hline Set & $\mathbf{L}_{\mathbf{1}}$ & $\mathbf{L}_{\mathbf{2}}$ & $\mathbf{L}_{\mathbf{3}}$ & $\mathbf{L}_{\mathbf{4}}$ \\
\hline 1 & 2.025 & 2.164 & 1.920 & 2.164 \\
\hline \hline
\end{tabular}

group. A previous study has shown a slight increase in the time to complete the task with age [6].

\section{Southampton Hand Assessment Procedure}

The Southampton Hand Assessment Procedure (SHAP) determines the effectiveness of a terminal device in grasp and manipulation by evaluating unilateral upperlimb performance. The SHAP consists of 26 timed tasks: 12 tasks involve grasping abstract objects such as spheres and prisms and 14 tasks simulate ADL. The tasks are performed by seated subjects in a standard order under the observation of an assessor, who records time to completion. The SHAP is designed to be a standardized and objective method of evaluating pathological hand function. The SHAP has undergone validation and reliability studies that support its use as an objective assessment tool [5]. However, this may be its first use in the evaluation of prosthetic hooks.

Light divided the 14 ADL into 6 prehensile patterns: $\mathrm{A}=$ spherical grip, required in 10 percent of the tasks; $\mathrm{B}=$ tripod pinch grip, required in 10 percent; $\mathrm{C}=$ power grip, also known as cylindrical or hook grip, required in 25 percent; $\mathrm{D}=$ lateral pinch grip, required in 20 percent; $\mathrm{E}=$ tip pinch grip, required in 20 percent; and $\mathrm{F}=$ extension grip, required in 10 percent [5]. Thus, a full range of natural grips is evaluated by the SHAP tasks, which are listed in Table 2. For these tasks [5], the opposite hand acts only as a stabilizer, thereby ensuring the functional assessment of the hooks.

\section{Procedures}

The subjects were asked to perform the SHAP tasks using their nondominant hand, a standard hook (Hosmer; Campbell, California) (Figure 2), and an adapted hook with the four-bar linkages attached (Figure 3). Each task was performed three times. The subjects were allowed a few minutes to familiarize themselves with each condition and to practice each task before being timed. The detailed protocol is reported by Light [5]. The normalhand results were used to obtain the normative data for the test. A boundary condition of eight times that of the average time, $\bar{x}_{i}$, for normal hands to perform a task was 
Table 2.

Subjects' completion times (mean \pm standard deviation) on Southampton Hand Assessment Procedure (SHAP) tasks using their nondominant anatomical hands.

\begin{tabular}{cllc}
\hline $\begin{array}{c}\text { Task } \\
\text { No. }\end{array}$ & Grip Type & \multicolumn{1}{c}{ SHAP Task } & $\begin{array}{c}\text { Completion } \\
\text { Time (s) }\end{array}$ \\
\hline 1 & A & Lightweight spherical & $1.55 \pm 0.54$ \\
2 & B & Lightweight tripod & $1.57 \pm 0.45$ \\
3 & C & Lightweight power & $1.50 \pm 0.34$ \\
4 & D & Lightweight lateral & $1.69 \pm 0.32$ \\
5 & E & Lightweight tip & $1.60 \pm 0.34$ \\
6 & F & Lightweight extension & $1.87 \pm 0.50$ \\
7 & A & Heavyweight spherical & $1.66 \pm 0.50$ \\
8 & B & Heavyweight tripod & $1.41 \pm 0.36$ \\
9 & C & Heavyweight power & $1.62 \pm 0.45$ \\
10 & D & Heavyweight lateral & $1.73 \pm 0.41$ \\
11 & E & Heavyweight tip & $1.56 \pm 0.43$ \\
12 & F & Heavyweight extension & $1.91 \pm 0.47$ \\
13 & E & Pick up coins & $4.94 \pm 0.82$ \\
14 & B and E & Undo buttons & $6.64 \pm 0.82$ \\
15 & B and C & Simulate food cutting & $4.50 \pm 1.58$ \\
16 & F & Simulate page turning & $1.87 \pm 0.47$ \\
17 & A & Remove jar lid & $2.24 \pm 0.55$ \\
18 & D & Pour water from jug & $4.90 \pm 0.99$ \\
19 & A & Pour water from carton & $8.00 \pm 1.25$ \\
20 & C & Move jar full of water & $2.08 \pm 0.50$ \\
21 & C & Move empty tin & $1.72 \pm 0.45$ \\
22 & D and F & Move tray & $3.39 \pm 0.66$ \\
23 & D and E & Turn key & $1.47 \pm 0.33$ \\
24 & D and E & Open/close zipper & $2.45 \pm 0.83$ \\
25 & C & Rotate screw & $3.73 \pm 0.95$ \\
26 & C & Turn door handle & $1.51 \pm 0.43$ \\
\hline *A spherical grip, B = tripod pinch grip, C = power grip (also known as \\
cylindrical or hook grip), D = lateral pinch grip, E = tip pinch grip, F = exten- \\
sion grip. & & & \\
\hline \hline & & & \\
\hline
\end{tabular}

imposed during hook testing to prevent a subject from taking too long [5]. This definite, if arbitrary, limit on the time allowed to perform a task provides a numerical value that can be used when subjects are unable to perform a task.

Time measurement for each task began when the subject pressed the button on the SHAP stopwatch. For tasks 1 to 12 (Table 2), subjects picked up each abstract object from the SHAP board, moved it through an obstacle, and placed it on the SHAP board; the opposing hand was only used in tasks 14 and 17, as specified by the SHAP protocol, to stabilize the grasped objects. For tasks 13 to 26, subjects simulated different ADL (Table 2). Time measurement for each task ended when the object was placed on the SHAP board and the button on the

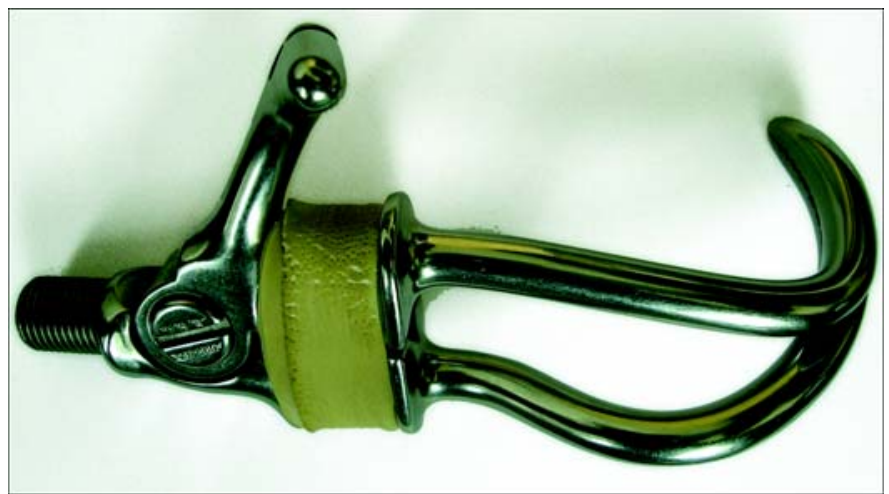

Figure 2.

Hosmer hook (Hosmer; Campbell, California).

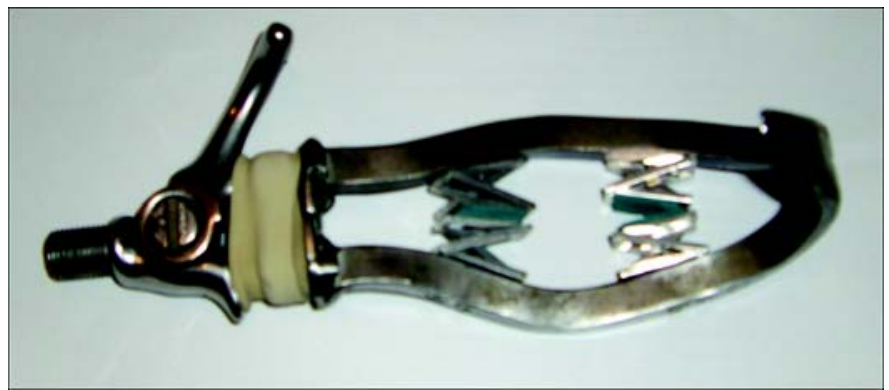

Figure 3.

Adapted hook with four-bar linkages attached.

SHAP stopwatch was pressed. The time was then read by the participant and recorded by the assessor. The SHAP's self-timed nature eliminates the need for subjective assessor opinion. The Index of Functionality (IOF) score given by the SHAP test is a normalized percentage [7]: 100 percent indicates normal hand function and 0 percent indicates minimal hand function, with minimal specified by the SHAP protocol as eight or more times slower than normal and normal determined by the subjects' average performance, $\bar{x}_{i}$, on the tasks with their healthy nondominant hand. The minimal function cutoff value permits data aggregation by making a necessary, if slightly arbitrary, value judgment as a compromise. Providing a standard time for the uncompleted tasks avoids analysis of only the completed tasks as an indication of the person's hand function. In addition, a floor effect caused by the uncompleted tasks is avoided. On the other hand, arbitrary large values assigned to incomplete tasks may decrease the sensitivity of the SHAP on tests that are completed. 
The total IOF score is made up of six subscores for each of the different hand grips: lateral, power, tripod, tip, extension, and spherical. The IOF is intended to distinguish between levels of function and may be obtained for each task and aggregated to describe prehensile patterns, the degree of use of the four bars, and the overall relative function of the two hooks as compared with a normal hand.

Some modifications, as given in Table 3, were made to the standard SHAP protocol to permit task completion with the prosthetic hooks.

\section{Statistical Test}

A statistical test of significance, the McNemar test of symmetry, was used to compare the success rates of the Hosmer hook and the hook with four bars.

\section{RESULTS}

The median, inner quartiles, minimum, and maximum normalized times for the Hosmer hook, the adapted hook, and the anatomical hand are shown in Figures 4 and 5.

Because our objective was to determine the benefit or detriment associated with the use of the four-bar mechanisms, we noted when the grasping strategies employed by the subjects utilized the four bars and when they did not. Because the four bars were attached proximally on the hooks and small objects were grasped distally, tasks involving the manipulation of small objects tended not to involve the four bars. Conversely, tasks requiring the

\section{Table 3.}

Modifications to standard Southampton Hand Assessment Procedure for prosthetic hook testing.

\begin{tabular}{cc}
\hline Task No. & \multicolumn{1}{c}{ Modification } \\
\hline $5,6,11,12,18-21$ & $\begin{array}{l}\text { Subjects could move form board to left or } \\
\text { right, as needed, to perform task. }\end{array}$
\end{tabular}

4, 10 These objects, thin rectangular prisms with a small handle, rotated and fell when handle was grasped with hook. Thus, they were often lifted by grasping prism instead of handle.

16

In picking up index card, subjects had to drag it, using hook, past edge of form board. manipulation of large objects always involved the four bars. Curiously, in some tasks, differences existed between users as to whether the four bars were used or not. The tasks are categorized by the degree of four-bar use in Figure 6.

The overall IOF was 65.75 (range: 56.93-78.61) for the Hosmer hook and 66.05 (range: 56.47-75.79) for the adapted hook with the four bars. One caveat in interpreting the IOF is that some of the tasks could not be completed by some subjects. This increases the timing (because the boundary condition of eight times the mean normative value is used) and decreases the IOF.

The IOF for each prehensile pattern, shown in Table 4, is calculated from the abstract objects listed in Figure $\mathbf{4}$ and the ADL listed in Figure 5, which are grouped into prehensile patterns according to the "natural" grip classification given in Table 2. In the spherical and power grasps, the adapted hook had a better average IOF. This result is in part due to the higher completion frequency with these grasps. Conversely, in the tripod, lateral, tip, and extension grasp patterns, the adapted hook had a lower IOF. In many of these tasks, the linkages were not used.

For the tasks in which 100 percent of the subjects used the four bars, the mean IOF was 72.06 for the Hosmer hook and 79.40 for the adapted hook. Table 5 shows that with the adapted hook, subjects were able to perform 10 of 11 tasks in less time, the exception being moving a tray. The largest improvements with the adapted hook were in the tasks heavyweight spherical, heavyweight power, removing the jar lid, and pouring water from a jug.

The mean IOF in the tasks for which 0 percent of subjects used the four bars (Table 6) shows the importance of the shape and material of the hook. For the tasks page-turning, turn a key, and open/close a zipper, the subjects used the inner side and tips of the hooks. The significant difference in the task open/close a zipper occurred because the adapted hook does not have high-friction material on the inner side of the hook and the Hosmer hook has a rubber coating on the tips and sides.

For the tasks listed in Table 7, some of the subjects used the four bars when using the adapted hook. Using the adapted hook, subjects were able to perform only three of nine of this subset of tasks in less time than when using the Hosmer hook.

Table 8 records activities with unsuccessfully completed tasks. We determined the statistical significance of the relative success rates with the standard and the 


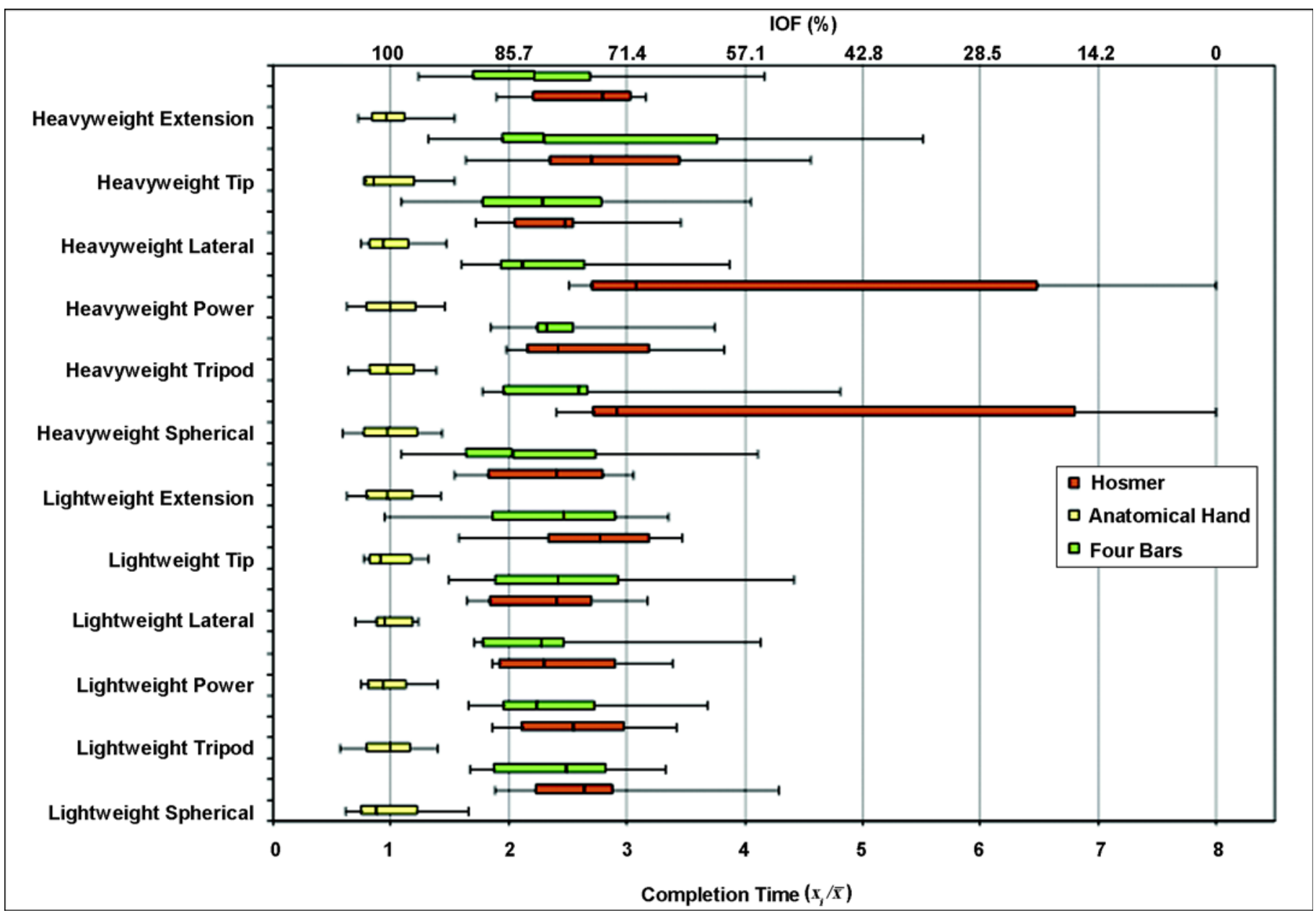

Figure 4.

Abstract objects: Normalized comparison of mean times and lower and upper times for standard Hosmer hook and adapted hook with four bars IOF = Index of Functionality, $\mathbf{x}_{\mathbf{i}} / \overline{\mathbf{x}}=$ task time using prosthesis divided by time using healthy hand.

adapted hooks by pairing each Hosmer-hook test with a corresponding test using the adapted hook. For each of the 10 subjects, the first Hosmer-hook test was paired with the first adapted-hook test; the second and third tests were likewise paired. For the 30 paired attempts at each task, a success with the standard (Hosmer) hook was designated $\mathrm{H}_{0}$ and a success with the adapted hook was designated $\mathrm{H}_{1}$; unsuccessful attempts were designated $\sim \mathrm{H}_{0}$ and $\sim \mathrm{H}_{1}$ for the standard and the adapted hooks, respectively. The pairs of tests were categorized according to whether both were successful $\left(\mathrm{H}_{0}\right.$ and $\left.\mathrm{H}_{1}\right)$, the standard was successful and the adapted was not $\left(\mathrm{H}_{0}\right.$ and $\left.\sim \mathrm{H}_{1}\right)$, the standard was not successful and the adapted was $\left(\sim \mathrm{H}_{0}\right.$ and $\left.\mathrm{H}_{1}\right)$, or neither was successful $\left(\sim \mathrm{H}_{0}\right.$ and $\left.\sim \mathrm{H}_{1}\right)$. Performing the McNemar test on the pair categorizations indicated that the different completion rates were statistically significant for each task in which noncompletions occurred. The inferences, shown in Table 8, indicate that for the tasks with large objects, the adapted hook performed better, with a statistical confidence level of 95 to 99 percent. Conversely, for the tasks undo buttons, simulate food cutting, and open/close a zipper, the standard hook performed better, with a statistical confidence level of 95 to 99 percent.

\section{DISCUSSION}

As noted previously, the SHAP test calls for a boundary condition to be imposed whenever a test goes 


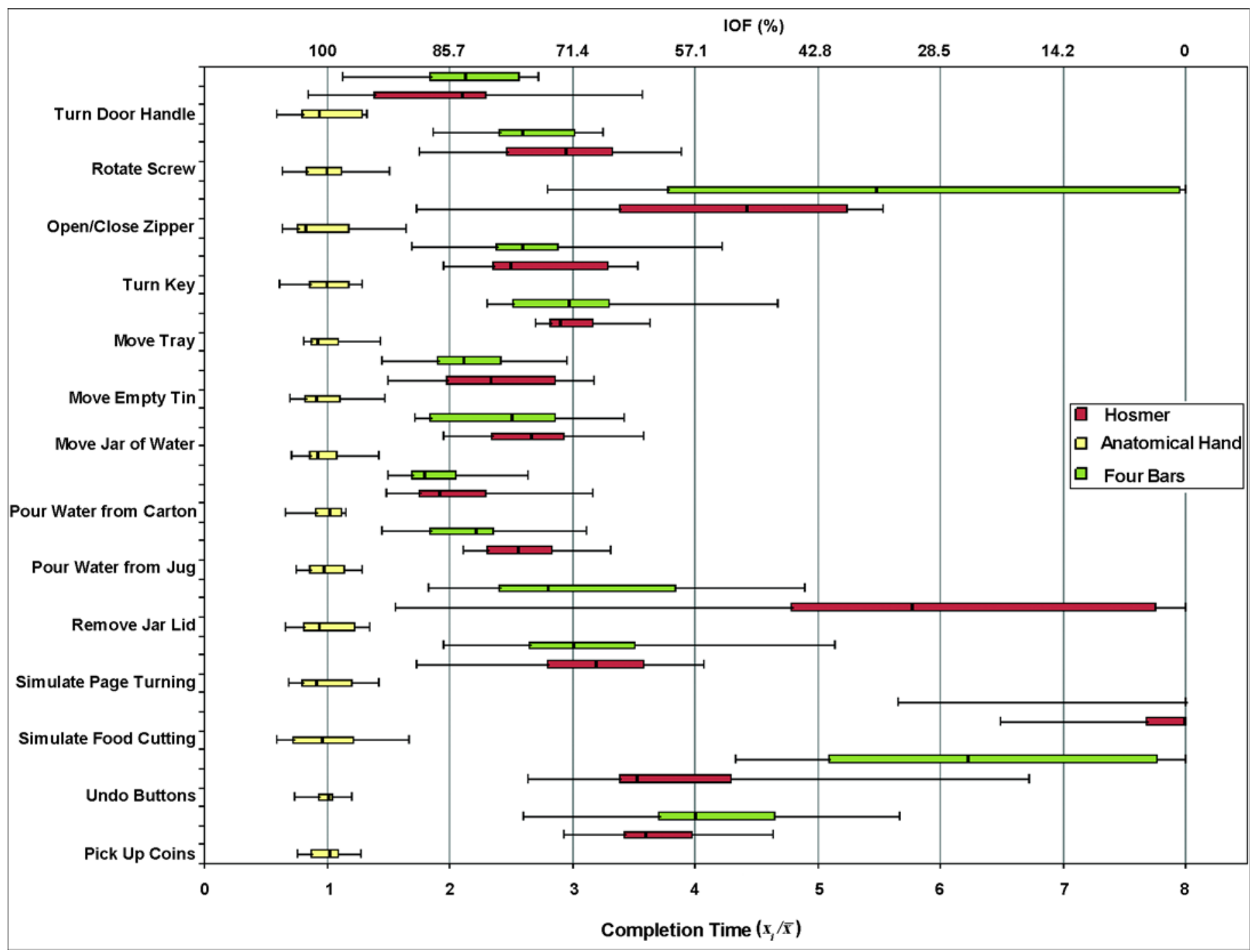

Figure 5.

Activities of daily living: Normalized comparison of mean times and lower and upper times for standard Hosmer hook and adapted hook with four bars. IOF = Index of Functionality, $\mathbf{x}_{i} / \overline{\mathbf{x}}=$ task time using prosthesis divided by time using healthy hand.

beyond eight times the mean normative time for the task. Noncompletion of a task poses a statistical dilemma because the basis for comparison, i.e., the time for completion, is undefined for those tasks. Conversely, differences in the completion rates between the two different hooks are a key indication of improvement or deterioration of function.

The adapted hook is an improvement for tasks requiring the manipulation of large, heavy, and round objects. Unfortunately, our current design does not incorporate the four bars at the distal end of the hook and so our results on grasping tasks involving small objects and pre- cision grasps support the use of a high-friction tip but indicate nothing about the use of four-bar passive surfaces at the distal end of a hook.

Our results are comparable to those of Gilad, who studied prosthetic-hook design and broke the manipulation of an object into five different components: reach, grasp, movement, position, and release [8]. His results indicated that the grasp portion of object manipulation was the major contributor to longer manipulation times in people with amputations. He also proposed a hook redesign based on the standard lyre-shaped hook, which offered improved grasp for spherical or ball-shaped 


\begin{tabular}{|c|c|c|}
\hline $\begin{array}{c}\text { Four Bars Used by } \\
100 \% \text { of Subjects }\end{array}$ & $\begin{array}{l}\text { Four Bars Used by } \\
\text { Some Subjects (\%) }\end{array}$ & $\begin{array}{l}\text { Four Bars Used by } \\
0 \% \text { of Subjects }\end{array}$ \\
\hline $\begin{array}{l}\text { - Lightweight Spherical } \\
\text { - Lightweight Power } \\
\text { - Heavyweight Spherical } \\
\text { - Heavyweight Power } \\
\text { - Remove Jar Lid } \\
\text { - Pour Water from Jug } \\
\text { - Pour Water from Carton } \\
\text { - Move Jar Full of Water } \\
\text { - Move Empty Tin } \\
\text { - Rotate Screw } \\
\text { - Move Tray }\end{array}$ & $\begin{array}{l}\text { - Lightweight Tripod (60) } \\
\text { - Lightweight Lateral (40) } \\
\text { - Heavyweight Tripod (30) } \\
\text { - Heavyweight Lateral (60) } \\
\text { - Heavyweight Tip }(40) \\
\text { - Heavyweight Extension (30) } \\
\text { - Pick Up Coins (20) } \\
\text { - Undo Buttons (10) } \\
\text { - Simulate Food Cutting (10) }\end{array}$ & $\begin{array}{l}\text { - Lightweight Tip } \\
\text { - Lightweight Extension } \\
\text { - Simulate Page Turning } \\
\text { - Turn Key } \\
\text { - Open/Close Zipper } \\
\text { - Turn Door Handle }\end{array}$ \\
\hline
\end{tabular}

Figure 6.

Variation of four-bar linkage use by task.

Table 4.

Prehensile-pattern average, minimum, and maximum Index of Functionality for standard Hosmer hook and adapted hook with four bars.

\begin{tabular}{|c|c|c|c|c|c|c|}
\hline \multirow{2}{*}{ Prehensile Pattern } & \multicolumn{3}{|c|}{ Hosmer Hook } & \multicolumn{3}{|c|}{ Hook with Four Bars } \\
\hline & Average & Minimum & Maximum & Average & Minimum & Maximum \\
\hline Spherical & 61.2 & 38.8 & 86.2 & 78.2 & 61.7 & 85.9 \\
\hline Tripod & 54.1 & 42.6 & 59.8 & 46.8 & 30.6 & 54.0 \\
\hline Lateral & 73.3 & 65.0 & 85.3 & 70.0 & 58.2 & 83.2 \\
\hline Tip & 66.4 & 50.8 & 77.8 & 56.8 & 37.5 & 73.8 \\
\hline
\end{tabular}

Table 5.

Task-specific average Index of Functionality during activities in which 100 percent of subjects used the four bars on adapted hook.

\begin{tabular}{lcc}
\hline \multicolumn{1}{c}{ Task } & Hosmer Hook & Hook with Four Bars \\
\hline Lightweight Spherical & 75.00 & 79.41 \\
Lightweight Power & 47.00 & 80.32 \\
Heavyweight Spherical & 78.99 & 76.29 \\
Heavyweight Power & 51.93 & 80.72 \\
Remove Jar Lid & 50.59 & 70.28 \\
Pour Water From Jug & 32.76 & 83.60 \\
Pour Water From Carton & 77.17 & 87.01 \\
Move Jar Full of Water & 84.86 & 79.73 \\
Move Empty Tin & 76.05 & 83.71 \\
Move Tray & 80.48 & 70.89 \\
Rotate Screw & 71.21 & 76.77 \\
Ensemble Average & 73.29 & 79.40
\end{tabular}

objects. Our results may also be compared with those of Light, who performed SHAP evaluation on three young (16-18 years old) subjects with amputations using myo- electric hands [5]. Their overall IOFs using the hands were 48.07, 42.73, and 37.02. The three subjects had prehensile pattern scores that were generally less than those 
Table 6.

Task-specific average Index of Functionality during activities in which 0 percent of subjects used the four bars on adapted hook.

\begin{tabular}{lcc}
\hline \multicolumn{1}{c}{ Task } & Hosmer Hook & Hook with Four Bars \\
\hline Lightweight Tip & 75.17 & 81.08 \\
Lightweight Extension & 81.10 & 82.56 \\
Simulate Page Turning & 69.54 & 69.61 \\
Turn Key & 75.48 & 74.70 \\
Open/Close Zipper & 55.76 & 32.82 \\
Turn Door Handle & 85.17 & 84.08 \\
\hline Ensemble Average & 74.74 & 74.24 \\
\hline \hline
\end{tabular}

Table 7.

Task-specific average Index of Functionality during activities in which some subjects used the four bars on adapted hook.

\begin{tabular}{lcc}
\hline \multicolumn{1}{c}{ Task } & Hosmer Hook & Hook with Four Bars \\
\hline Lightweight Tripod & 77.54 & 80.06 \\
Lightweight Lateral & 80.78 & 78.25 \\
Heavyweight Tripod & 76.09 & 77.85 \\
Heavyweight Lateral & 79.78 & 79.72 \\
Heavyweight Tip & 72.84 & 72.42 \\
Heavyweight Extension & 76.81 & 81.39 \\
Pick Up Coins & 61.26 & 55.27 \\
Undo Buttons & 58.16 & 24.80 \\
Simulate Food Cutting & 4.93 & 4.58 \\
Ensemble Average & 69.54 & 67.83 \\
\hline \hline
\end{tabular}

Table 8.

Frequencies of task completion and noncompletion for standard and adapted hooks.

\begin{tabular}{lccccc}
\hline \multicolumn{1}{c}{ Task } & $\mathbf{H}_{\mathbf{0}}$ and $\mathbf{H}_{\mathbf{1}}$ & $\mathbf{H}_{\mathbf{0}}$ and $\sim \mathbf{H}_{\mathbf{1}}$ & $\sim \mathbf{H}_{\mathbf{0}}$ and $\mathbf{H}_{\mathbf{1}}$ & $\sim \mathbf{H}_{\mathbf{0}}$ and $\sim \mathbf{H}_{\mathbf{1}}$ & Significance (\%) \\
\hline Heavyweight Spherical & 21 & 0 & 9 & 0 & 0 \\
Heavyweight Power & 21 & 0 & 9 & 0 & 0 \\
Undo Buttons & 21 & 9 & 0 & 18 & 99 \\
Simulate Food Cutting & 6 & 6 & 6 & 0 & 99 \\
Remove Jar Lid & 24 & 0 & 0 & 0 & 95 \\
Open/Close Zipper & 24 & 6 & 95
\end{tabular}

$\mathrm{H}_{0}=$ successful task completion with standard hook, $\mathrm{H}_{1}=$ successful task completion with adapted hook, $\sim \mathrm{H}_{0}=$ unsuccessful task completion with standard hook, $\sim \mathrm{H}_{1}=$ unsuccessful task completion with adapted hook.

given in Table 4; i.e., spherical: 80, 55, 37; tripod: 45, 35, 30; power: 55, 30, 30; lateral: 50, 35, 50; tip: 20, 35, 45; and extension: 60, 55, 35. These observations accord with those of Stein and Walley that myoelectric hand users take six times as long to perform a task (IOF: 30) and hook users take three times as long (IOF: 70) as those with natural hand function [6].

This work indicates that improvement in grasp function is possible with the use of passive fingertip mechanisms. In particular, we note that at some tasks, an exceptional user of the adapted hooks could achieve performance times comparable with those achieved with the anatomical hands. Because work by Miller et al. indicates that prosthetic control deteriorates even with electromyography in systems with more than two degrees of freedom [9], passive grasping systems, such as these four-bar linkages, are likely to continue to have a role in improving prosthetic function.

Uncontrolled variables in the testing were-

1. Individual attributes of the subjects: Some seemed to be quick learners and were able to perform the tasks with relative ease. Upper-body strength also seemed to help some subjects open the hook faster. Stronger subjects were also able to use two rubber bands, while 
weaker subjects used only one, causing a disparity in prosthetic grip force.

2. Frustration: Some subjects became frustrated at being unable to complete tasks. Scores for difficult ADL, such as picking up coins, undoing buttons, simulating food cutting, pouring water, and opening/closing a zipper, may have been affected by subjects becoming frustrated.

3. Rubber bands: The rubber bands, which provide passive closing of the hooks, were changed after every two subjects to reduce the bands' tendency to degrade over time.

4. Hook order: Five subjects used the standard Hosmer hook first and five used the adapted hook first. Their scores using their second type of hook may have been improved because of increased experience using a terminal device.

Future research should expand on the findings of the current study. The within-subject research design in a controlled environment provides an excellent opportunity to minimize the variability between subjects and focus on the differences between prosthetic mechanics. Controlling for aspects of the terminal device other than the experimental mechanism allows for more critical analysis of its beneficial or detrimental aspects. Similar controlled experiments may allow some aspects of terminal devices to be optimized for a given set of conditions. However, the results of these experiments should be expanded to include actual ADL and subjects with amputations.

\section{CONCLUSIONS}

In testing, heavyweight power and spherical grasps were improved by the use of a passive four-bar mechanism at the point of contact. Conversely, precision grasps were worsened, not because of the four-bar adaptation but because of the lack of a high-friction surface on the distal end of our prototype. Further research is needed to determine improvement or detriment in precision grasps with four bars attached to the distal ends of hooks. We recommend that future prototypes have the oval opening or lyre-shape characteristic of the Hosmer hook and highfriction surfaces on the distal tips. We also believe that some degree of passive adjustability to the shape of the grasped object is desirable.

\section{ACKNOWLEDGMENTS}

Author Contributions:

Acquisition of data: I. A. Ramirez.

Analysis and interpretation of data: I. A. Ramirez, C. P. Lusk.

Drafting of manuscript: I. A. Ramirez.

Statistical analysis: I. A. Ramirez.

Critical revision of manuscript for important intellectual content:

C. P. Lusk, M. J. Highsmith, M. E. Maitland.

Study supervision: C. P. Lusk.

Study concept and design: R. Dubey, M. E. Maitland.

Administrative, technical, or material support: R. Dubey, M. J. Highsmith, M. E. Maitland

Financial Disclosures: The authors have filed a patent application on the adapted hook design.

Funding/Support: Student funding for this research was provided by the National Science Foundation Florida-Georgia Louis Stokes Alliance for Minority Participation program Bridge to the Doctorate supplement award to the University of South Florida (HRD 0217675). The funding grant "Demonstration project in prosthetics and orthotics" is issued by the Department of Education/Rehabilitation Services Administrationfunded project 6140100000 .

Participant Follow-Up: The authors do not plan to inform participants of the publication of this study because contact information is unavailable.

\section{REFERENCES}

1. Highsmith MJ, Carey SL, Koelsch KW, Lusk CP, Maitland ME. Kinematic evaluation of terminal devices for kayaking with upper extremity amputation. J Prosthet Orthot. 2007; 19(3):84-90. DOI:10.1097/JPO.0b013e31806ada2f

2. TRS Complete Product Listing, 2009 [Internet]. Bouler (CO): TRS, Inc [cited 2007 May 19]. Available from: http://www.oandp.com/products/trs/products/.

3. Ramirez IA, Lusk CP, Maitland ME. Linkage-based prosthetic fingertips: Stability analysis. 2007 Florida Conference on Recent Advances in Robotics and Robot Showcase; 2007 May 31-Jun 1; Tampa, FL. Tampa (FL): University of South Florida; 2007. Available from:

http://fcrar2007.eng.usf.edu/Papers/ 13 Ramirez Lusk Maitland.pdf/.

4. Lake C. Effects of prosthetic training on upper-extremity prosthesis use. J Prosthet Orthot. 1997;9(1):3-9.

5. Light CM. An intelligent hand prosthesis and evaluation of pathological and prosthetic hand function [dissertation]. [Southampton (UK)]: University of Southampton Press; 2000.

6. Stein RB, Walley M. Functional comparison of upper extremity amputees using myoelectric and conventional prostheses. Arch Phys Med Rehabil. 1983;64(6):243-48. [PMID: 6860093]

7. Light CM, Chappell PH, Kyberd PJ. Establishing a standardized clinical assessment tool of pathologic and prosthetic hand function: Normative data, reliability, and 
JRRD, Volume 46, Number 8, 2009

validity. Arch Phys Med Rehabil. 2002;83(6):777-83.

[PMID: 12048655]

8. Gilad I. Motion pattern analysis for evaluation and design of a prosthetic hook. Arch Phys Med Rehabil. 1985;66(6): 399-402. [PMID: 4004541]

9. Miller LA, Lipschutz RD, Stubblefield KA, Lock BA, Haung H, Williams TW 3rd, Weir RF, Kuiken TA. Control of a six degree of freedom prosthetic arm after targeted muscle reinnervation surgery. Arch Phys Med Rehabil. 2008; 89(11):2057-65. [PMID: 18996233]

Submitted for publication January 22, 2009. Accepted in revised form June 5, 2009. 